\title{
An Optimal Method to Design Wireless Sensor Network Structures
}

\author{
https://doi.org/10.3991/ijoe.v14i01.8062 \\ Ling Yang $\left.{ }^{\varpi}\right)$, Xucan Cai, Chunlin Wu \\ Zhongkai University of Agriculture and Engineering, Guangzhou, China \\ k25820031zhangu@163.com
}

\begin{abstract}
In order to optimize the structure of wireless sensor network, an improved wireless sensor network sleep mechanism is proposed. First, some nodes in the area with too high redundancy are dormant by density control, so that the active nodes are even more distributed. Then, the active node is subjected to circular coverage redundancy decision. Different circumferential coverage decision methods are used for network boundary nodes and non-boundary nodes. As a result, the boundary nodes and non-boundary nodes are well dormant, and the network redundancy is reduced. The simulation results show that the improved dormancy mechanism makes the number of active nodes in the network smaller and more evenly, and the network lifetime is extended on the basis of maintaining the original coverage of the network. Therefore, the proposed method can achieve optimal coverage in wireless sensor networks. The network prolongs network lifetime while ensuring reliable monitoring performance.
\end{abstract}

Keywords-wireless sensor networks, dormancy mechanism, reconstruction, wake up, node mobility

\section{Introduction}

Wireless sensor network is the multi hop and self-organizing network system composed through wireless communication of a large number of nodes with a probability distribution model randomly deployed in the monitoring region [1]. Wireless sensor technology has been gradually extended from the earliest military defense applications to agriculture, environmental monitoring, biological medicine, traffic management, remote management of dangerous areas and even home and other fields [2-3]. For high density network application, there are a large number of dormant redundant nodes. We can design the redundant node identification algorithm, to make parts of the nodes have alternate dormancy without affecting the network coverage and connectivity requirements, thus reducing the flow for sending data flow to an important node, and saving the energy of essential nodes. For the important nodes, the energy consumption level is higher, able to dispenser the relay node around an important node and help the important node to share the task of transmitting large amount of data flow. In this way, it can guarantee the service life of an important node. The 
method of solving this problem at present mainly focuses on designing an effective MAC protocol, identifying the redundant nodes and making it dormant, so as to ensure prolonging the life cycle of important nodes and design a rational network topology and node rotation dormancy [4].

The sensor node uses a limited battery capacity. Taking a typical hardware platform as an example, two AAA batteries of one node can only work for 100 120 hours. Sensor networks have huge number of nodes, and are generally deployed in unattended harsh environment, so it is very difficult to supply power to nodes. Therefore, to meet the coverage and connectivity requirements, node energy consumption should be reduced to the maximum extent, thereby extending network life. At present, the mainstream solution to the coverage problem is to save energy consumption by scheduling node on-time. Part of the redundant nodes are temporarily closed, so that all nodes take turns dormancy, thereby enhancing energy efficiency. At present, the typical dormancy mechanisms of wireless sensor networks mainly include dormancy mechanism based on node density control, dormancy mechanism based on random sleep and dormancy mechanism based on redundant node determination. Among them, the sleep mechanism based on node density control and the sleep mechanism based on random sleep are suitable for occasions where the coverage requirement is not very high. The sleeping mechanism based on redundant node decision can provide full coverage of the target area.

In this paper, a new redundancy node decision algorithm is proposed to solve the above problems. Combined with the idea of circumferential coverage, an improved sleep mechanism of wireless sensor network is designed. The algorithm first performs a density control on the network to make some nodes in the over-redundant area sleep. Then, based on the circumferential coverage, other nodes are subjected to redundancy determination. The judgment of the boundary node is different from that of the non-boundary node. The improved sleeping mechanism is proposed on the basis of the improved redundancy node decision algorithm. The remaining energy of the node is taken as a priority. It prevents redundant nodes and shuts down the coverage dead zone in the network at the same time.

\section{State of the art}

As early as the 70 s of last century, point to point transmission was introduced to traditional sensors [7]. The sensor network prototype formed by connecting the sensor controller. With the continuous development and progress of related disciplines, the sensor network also has the comprehensive processing ability to obtain a variety of information signals. By associating with the sensor controller, a sensor network with information synthesis and processing capability is formed. From the end of the last century, field bus technology began to be applied to the sensor network. People use it to build an intelligent sensor network. A large number of multi-function sensors are used and connected using wireless technology, and a wireless sensor network is gradually formed [8]. The dormancy mechanism of the wireless sensor network means that the redundant nodes are closed by controlling the working status of the sensor 
nodes, so as to prolong the life of the sensor network. The purpose is to improve the sensor network perception, monitoring, communications and other service quality. The sleep mechanism can be applied to nodes with the same function in the network. In addition, it can also be used in conjunction with hierarchical clustering topology control algorithms. Ye et al proposed a distributed node density control algorithm PEAS. The node is dormant in the initial state and is awakened after a random delay [9]. The PEAS algorithm is simple and does not depend on the location information of the nodes. The calculation overhead is small, but the coverage quality of the network cannot be guaranteed. Cerpa proposes ASCENT agreement. The ASCENT algorithm has good robustness and scalability. However, it cannot make the boundary nodes sleep on the target area, and cannot guarantee the uniform energy consumption.

For wireless sensor networks, it is two important goals to balance the energy consumption of the sensor nodes and improve the life cycle of the network. The distribution of sensor nodes in a topology affects the energy consumption of each node [10]. In the process of transmitting information, due to the different location of the node, the energy loss is also different. In 2009, Xu Gang et al. proposed application-level node dormancy / synchronization mechanism, and provided a method to calculate the empty proportion based on the expected lifetime and data acquisition requirements of nodes. The results show that the lifetime of the nodes is prolonged. With sleep technology, 150h of no sleep node life can reach more than 1 year. In 2013, Hong Lu et al. proposed a dormancy algorithm for underwater sensor network nodes based on tree topologies. The algorithm can effectively shorten the number of wake-up times, prolong the sleep time, and ensure that the end-to-end propagation delay is not affected by the sleep time. In 2015, Chen Long and other proposed a comprehensive sensor network clustering and node sleep mechanism of the agreement. It divides the life of the sensor network into several time periods. In each time period, it determines the set of similar nodes for each node, and selects the representative node (Rnode) to send the perceived data. In addition, it also hides some redundant nodes. The results show that the protocol can extend the life of the sensor network. In 2016, Feng Lin proposed a border area deployment adjustment of hybrid sensor network node optimization algorithm. By adding a small number of mobile nodes to the divided boundary area, it achieves the energy balance in the process of node scheduling, and realizes the purpose of extending the network lifetime.

\section{$3 \quad$ Methodology}

\subsection{Assumptions of the algorithm}

In this paper, assuming that all nodes are randomly distributed uniformly in a square area, the node density is large enough. All nodes are able to cover the entire area while they are working. It is assumed that the network has the following properties:

First, each node can conduct a full range of exploration. The coverage area is a circular area centered on the node and the radius of the node detection distance $r_{s}$. 
Second, the communication radius of the node is $r_{c}=2 r_{s}$, so that the full coverage is guaranteed only when the full coverage is guaranteed.

Third, all nodes are isomorphic, that is, the detection radius $r_{s}$ is the same as the communication radius $r_{c}$.

Fourth, node uses Boolean perception model. All nodes within the perception range of each node can receive messages sent by them. However, the nodes outside the perceptual range cannot receive messages sent by them.

Fifth, nodes can be measured or positioned to obtain their specific location.

Sixth, all the sensor nodes in the target area are in the same two-dimensional plane, and the whole target area is regarded as a convex region.

\subsection{Network evaluation index}

This paper evaluates the advantages and disadvantages of wireless sensor network coverage optimization algorithm from the following aspects:

Coverage rate: the coverage capability of network is an important index to measure the performance of sleep mechanism. Network coverage is usually used to reflect the coverage capacity of the network. In general, it is defined as the ratio of the effective coverage area $A_{a}$ of the network to the area $A$ of the target area, that is:

$$
C_{a}=A_{a} / A=\bigcup_{i=1}^{n_{a}} S^{i} / A, i \in N_{a}
$$

Among them, $A_{a}$ is the union of all active nodes coverage. $S^{k}$ is the coverage of node $\mathrm{K} . \mathrm{N}_{\mathrm{a}}$ is a set of active nodes in the region.

Coverage efficiency: the coverage efficiency in dormancy mechanism is defined as the ratio of union of all activities coverage area and the sum of all active nodes coverage. That is:

$$
C_{e}=A_{a} / A_{s}=\bigcup_{i=1}^{n_{a}} S^{i} / \sum_{i=1}^{n_{a}} S^{i}, i \in N_{a}
$$

It is used to measure the utilization of nodes' perception range. On the one hand, it can reflect the situation of network coverage; on the other hand, it can reflect the energy consumption of the whole network. At the same time, the redundancy of active nodes is reflected, and the redundancy of active nodes is small when the coverage efficiency is high, otherwise the redundancy is large.

Number of active nodes: in the case of meeting the requirement of network coverage, the number of active nodes is less, the effective coverage area of nodes is larger, and the utilization ratio of nodes is higher, which can effectively reduce the energy consumption of the network.

Network lifetime: the hardware platform resource of sensor network node is limited, the number of network nodes is huge, and the environment condition of practical application does not allow battery replacement of "invalid" node. Therefore, the life- 
time of sensor networks is an important performance index for coverage optimization algorithms.

\subsection{Redundant coverage and related algorithms}

Because of the wireless sensor network node hardware platform with limited resources, huge amount of network nodes amount and complex practical application environmental conditions and not allowing to replace the battery of "failure" nodes, it is an effective measurement for saving the nodes with limited battery energy and trying to prolong the whole network by reducing the energy consumption of the nodes. It is implemented by reducing the redundant broadcasting amount and making more nodes in the dormant state in the case of ensuring complete coverage. The reason is that the energy consumption of wireless communication module nodes accounted for more than $90 \%$ of the total energy consumption, the wireless communication module has the maximum energy consumption in the sending state, followed by the receiving state and the idle state, and the energy consumption of dormant state is the lowest. At present, when the communication module of the commonly used Berkeley Motes sensor is in the transmitting state, the power consumption is $60 \mathrm{~mW}$, when in receiving state and idle state, the power consumption is $12 \mathrm{~mW}$, and in the dormant state, the power consumption is only $0.03 \mathrm{~mW}$. Therefore, it will make parts of the nodes in the dormant state and greatly reduce the energy consumption of the nodes and prolong the working time of the nodes under the premise of satisfying the coverage requirements.

The premise of the redundant node dormancy algorithm is the determination of redundant nodes, that is to say, the nodes judge whether the covered area can be completely detected by the neighbor nodes according to the information of neighbor nodes. After the judgment of redundant nodes, because it is a distributed algorithm, each node, only based on the neighbor information, judges whether it belongs to the node. However, since that the correlation of the node coverage is not that each node can be dormant, for example in Figure 1, node 5 and node 6 can determine itself as redundant nodes but not dormant at the same time of node 5 and node 6 , otherwise, between node 1, 2, 3 and 4, it will produce the coverage holes. Therefore, how to design the redundant node algorithm is an important part of the research on the coverage problem of wireless sensor networks. The coverage holes are as shown in Figure 1 .

At present, the main algorithms for judging redundant nodes are Di Tian algorithm [5] and CCP algorithm [6]. The study found that these algorithms have a certain degree of problems. In the following, we will analyze and elaborate in detail.

The idea of Di Tian algorithm is that if a node is covered by all the neighbor nodes $[0,2 \pi]$, then the node is a redundant node. Specific as follows: for any node $v$, define its neighbor node set $N(v)=\left\{u \mid d(u, v) \leq r_{S}\right\}, d(u, v)$ indicating the distance from $u$ to $v$. Define the open angle of the neighbor node $\mathrm{u}$ to $\mathrm{v}$ as $\beta_{\mathrm{ui} \rightarrow \mathrm{v}}$, as shown in Figure 2 $\angle P_{1} A P_{2}$. 

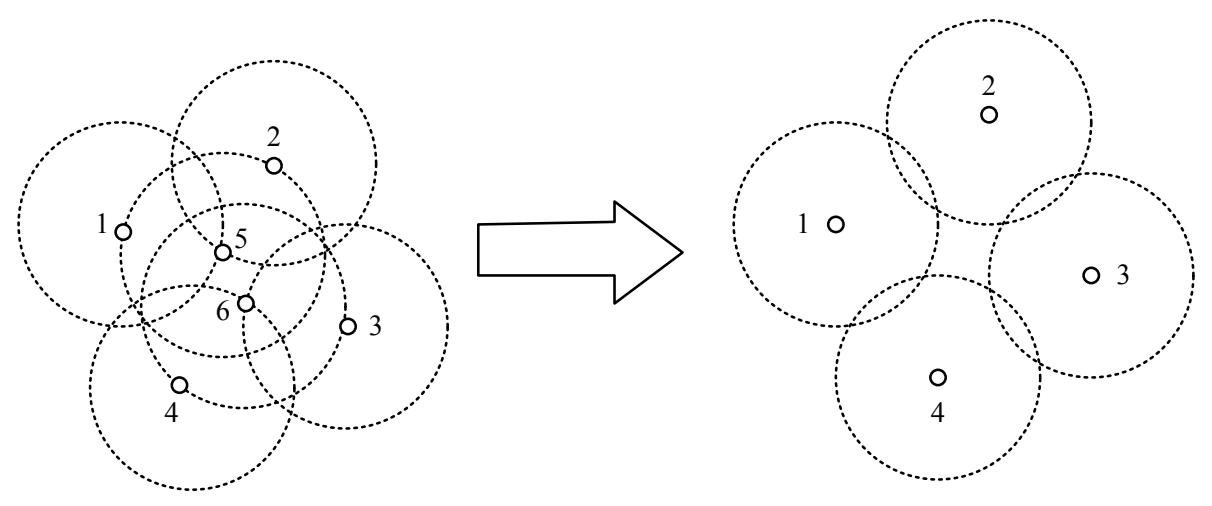

Fig. 1. Coverage holes

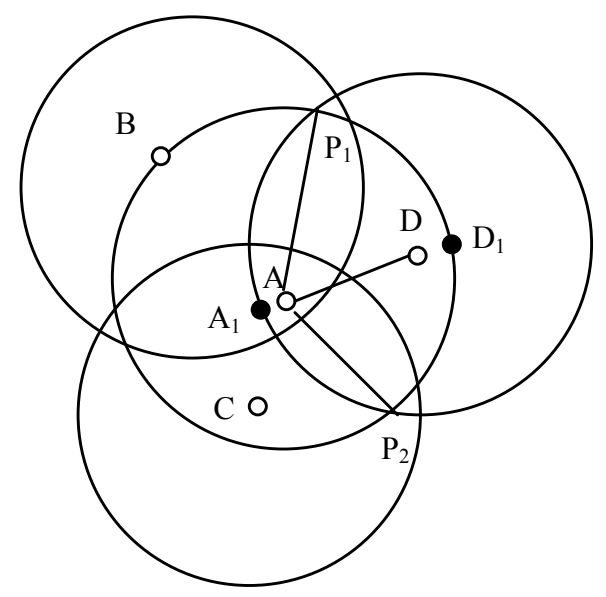

Fig. 2. Di Tian algorithm

If all the neighbor nodes of the node $\mathrm{v}$ to the set of the open angle of $\mathrm{v}$ covers $[0,2 \pi]$, that is $\cup \beta_{\mathrm{ui} \rightarrow \mathrm{v}}=\lfloor 0,2 \pi\rfloor$, then the node $\mathrm{v}$ is a redundant node.

$$
\mathrm{u}_{\mathrm{i}} \in N(v)
$$

In Figure 2, Node $A$ is covered by $B, C$, and $D$ because $\angle_{B \rightarrow A} \cup \angle_{C \rightarrow A} \cup \angle_{D \rightarrow A}=[0,2 \pi]$. In the Di Tian algorithm, because of using the opening angle to judge the coverage, it makes each node, in considering whether or not covered, only considers the nodes with the distance not greater than the radius of the probe, which makes the set of the redundant nodes calculated tends to be small.

In Figure 3, the area of node $\mathrm{A}$ is completely covered by $\mathrm{B}, \mathrm{C}$, and D. But $D \notin N(A)$, according to Di Tian algorithm, A cannot be covered by $\mathrm{B}$ and $\mathrm{C}$, so we determine that $\mathrm{A}$ is not redundant nodes, which makes the wrong judgment, and makes the finally judged redundant nodes are small in number. The wrong judgment in Di Tian algorithm is shown in Figure 3. 


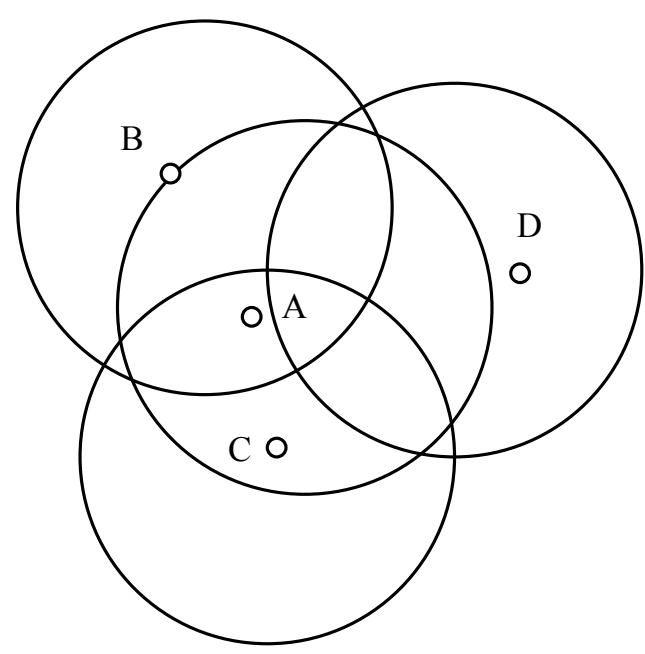

Fig. 3. Wrong judgment in Di Tian algorithm

The detection range of two nodes $\mathrm{u}$ and $\mathrm{v}$ is overlapped, and the condition needs to meet is that its distance is less than two times of the detection distance, namely $d(u, v) \leq 2 r_{S}$. In order to solve the problem of Di Tian algorithm, CCP algorithm defines the neighbor nodes set of any node $\mathrm{v}$ as $N(v)=\left\{u \mid d(u, v) \leq r_{S}\right\}$, which enlarges the search range and improves the accuracy of the judgment.

The judgment idea of CCP algorithm is based on a theory in the graph theory. That is to say, in the circle $\mathrm{C}(\mathrm{v})$ of taking node $\mathrm{v}$ as the center, and detection radius $\mathrm{r}_{\mathrm{s}}$ as the radius, the intersection of any two neighbor nodes is always covered by the third neighbor node, then the detection area of node $\mathrm{v}$ is covered by its neighbor nodes, that is, $\forall P \in[C(x) \cap C(y)], \exists \mathrm{z}$ and $P \in C(x)\{x, y, z\} \subseteq N(v)$. This algorithm does not have any error, and it will not judge redundant nodes with missing. The only problem is that the computational complexity is too high, analyzing as follows. It is assumed that the node $\mathrm{v}$ has $\mathrm{n}$ neighbor nodes, then the circle intersection of these neighbor nodes is up to $C_{\mathrm{n}}^{2}$ intersections, and the amount level is o $\left(n^{2}\right)$. The node v first of all calculates the node position of $\mathrm{o}\left(n^{2}\right)$, then conducts its traversal of $\mathrm{n}-2$ neighbor nodes, to determine whether the cross point is covered by the third-party node. Its computation complexity is $\mathrm{o}\left(n^{3}\right)$, and this calculation complexity in the high-density network is very high. For instance, $\mathrm{n}=20$, the computational complexity is reached o (8000) level, and the CPU computing capability of sensor nodes is limited, which makes the algorithm not applicable in wireless sensor networks. 


\section{$4 \quad$ Result analysis and discussion}

\subsection{Comparison of active nodes}

In the simulation, each experimental result is the average of 100 random topological experiments. Suppose that $r_{s}=6 m$, the total number of nodes $n$ respectively take $\{100,150,200,250,300\}$ five values. The number of active nodes under the two algorithms is observed with the total number of nodes. When $\mathrm{rs}=6 \mathrm{~m}$, the number of active nodes with the total number of nodes is as shown in Figure 4.

As can be seen from Figure 4, under the same conditions, the ECBNSS algorithm can put more redundant nodes into the sleep state, so that the nodes in active state are less. The number of active nodes calculated by Tian D algorithm increases linearly with the increase of $n$. However, the number of active nodes calculated by ECBNSS algorithm does not increase with the increase of $\mathrm{N}$, and always stays at about 60 . Table 1 is the statistical data of the number of active nodes varying with the total number of nodes under the two algorithms.

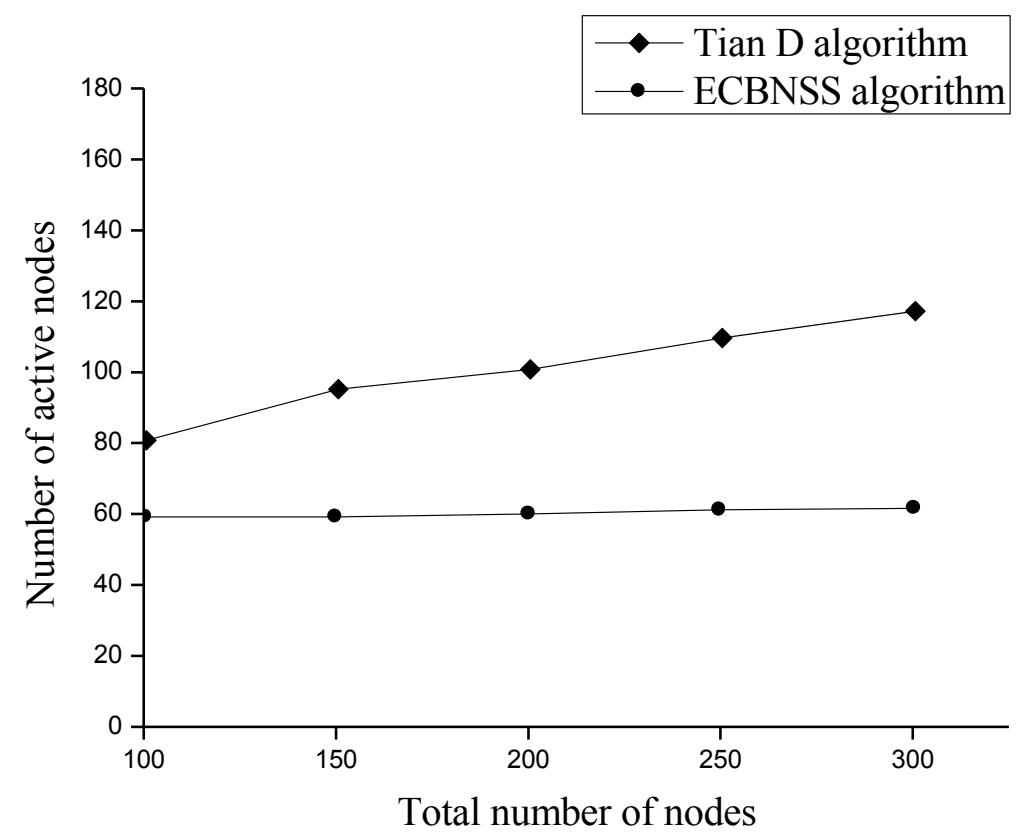

Fig. 4. Comparison of the number of active nodes

Table 1. Comparison of the number of active nodes

\begin{tabular}{|l|l|c|c|c|c|c|}
\hline \multicolumn{2}{|l|}{ Total number of nodes n } & 100 & 150 & 200 & 250 & 300 \\
\hline Number of & Tian D algorithm & 81 & 96 & 102 & 111 & 118 \\
\cline { 2 - 7 } active nodes & ECBNSS algorithm & 59 & 60 & 61 & 62 & 62 \\
\hline The proportional reduction & $27.2 \%$ & $37.5 \%$ & $40.2 \%$ & $44.1 \%$ & $47.5 \%$ \\
\hline
\end{tabular}


According to the simulation results, the $100 \mathrm{r}_{\mathrm{s}}=6 \mathrm{~m}$ nodes is deployed in the network. After running the Tian D algorithm, 81 nodes are in the working state. However, the ECBNSS algorithm only requires 59 nodes to work, and reduces the active nodes by $27.2 \%$. When 300 nodes with $r_{s}=6 \mathrm{~m}$ are scattered in the network, the number of active nodes in Tian D algorithm is increased by 37 compared with that in 100 nodes. The ECBNSS algorithm only adds 3, and it doesn't change much. With the increase of the number of nodes, the superior performance of ECBNSS algorithm becomes more obvious.

$\mathrm{n}=200, \mathrm{r}_{\mathrm{s}}$ respectively take $\{6,8,10,12\}$ four values. The number of active nodes under the two algorithms is observed as the change of perceived radius. When $n=200$, the number of active nodes varies with the node perception radius, as shown in Figure 5 .

As can be seen from Figure 5, the number of active nodes in both algorithms decreases with the increase of $r_{s}$, and the trend of change is not much different. The difference between the two $r_{s}$ is little. Table 2 shows the statistics of the change of perceived radius with the number of active nodes under the two algorithms.

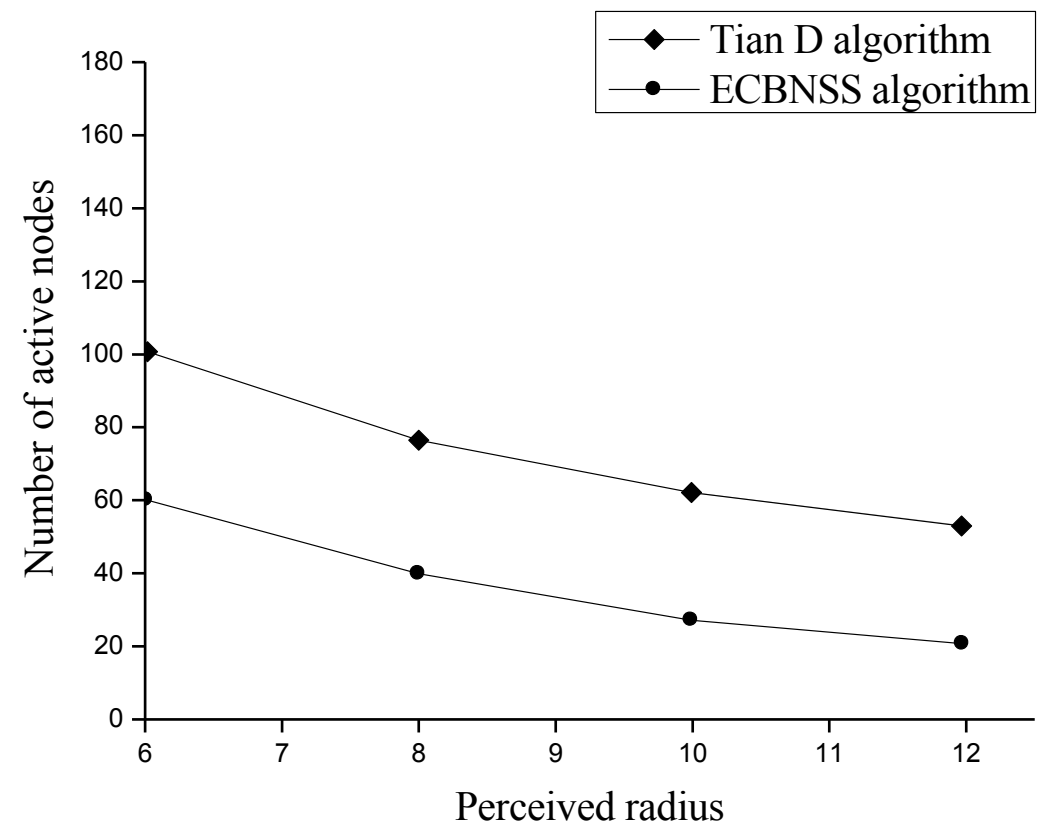

Fig. 5. Comparison of the number of active nodes

Table 2. Comparison of the number of active nodes

\begin{tabular}{|l|l|c|c|c|c|}
\hline Perceived radius $\mathbf{r}_{\mathbf{s}} / \mathbf{m}$ & 6 & 8 & 10 & 12 \\
\hline \multirow{2}{*}{$\begin{array}{l}\text { Number of } \\
\text { active nodes }\end{array}$} & Tian D algorithm & 102 & 77 & 62 & 53 \\
\cline { 2 - 6 } & ECBNSS algorithm & 61 & 41 & 27 & 20 \\
\hline \multicolumn{2}{|l|}{ The proportional reduction } & $41 \%$ & $36 \%$ & $35 \%$ & $33 \%$ \\
\hline
\end{tabular}


According to the simulation results, when $r_{s}=6 \mathrm{~m}, 102$ nodes are in working state after running Tian D algorithm. The ECBNSS algorithm only needs 61 nodes to work, and reduces the active nodes by $41 \%$. When $r_{s}=12 \mathrm{~m}$, the number of active nodes in Tian D algorithm is 53. There are only 20 active nodes in the ECBNSS algorithm, which reduces the active nodes by $33 \%$. It can be seen that the ECBNSS algorithm shows superior performance under various perceived radius.

From the above simulation results, it can be seen that the number of active nodes in ECBNSS algorithm does not increase with the increase of the sum of points, but basically maintains stability. The number of active nodes is much less than that of Tian D algorithm in the total number of nodes and detection radius. Therefore, the ECBNSS algorithm can control the number of nodes and prolong the lifetime of the network.

\subsection{Comparison of coverage efficiency}

Both the ECBNSS algorithm and the Tian D algorithm can guarantee full coverage, and the coverage rate of the simulation is approximately 1 . Therefore, coverage efficiency is compared. Coverage efficiency is the ratio of the union of coverage of all active nodes in a zone to the sum of coverage of all active nodes. This indicator reflects the redundancy of active nodes. Figure 6 shows the comparison of network coverage efficiency of the two algorithms when $r_{s}=10 \mathrm{~m}$.

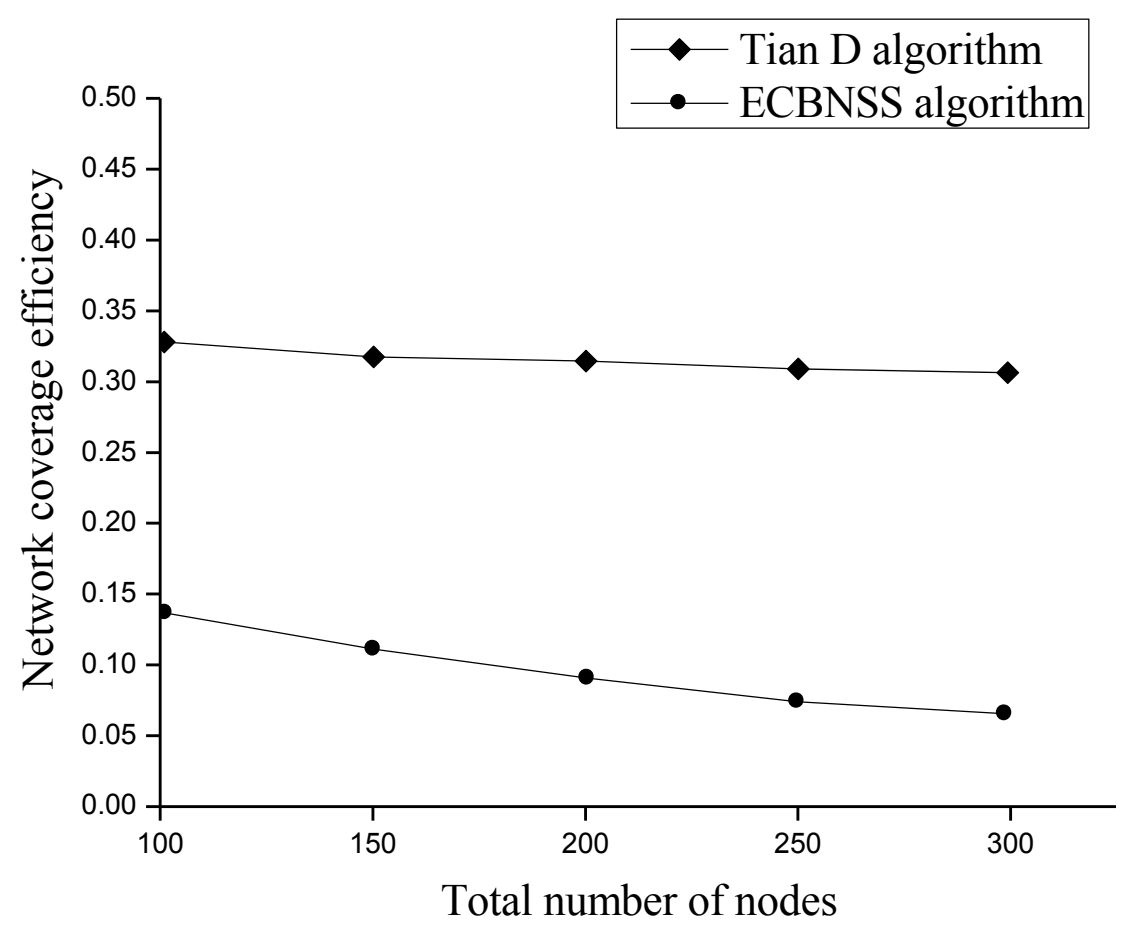

Fig. 6. Relationship between the total number of nodes and network coverage efficiency 
As can be seen from Figure 6, although both algorithms can guarantee full coverage, the node utilization rate of Tian D algorithm is apparently lower than that of ECBNSS algorithm. Especially when the node density increases, the coverage efficiency of Tian D algorithm decreases greatly, while the coverage efficiency of ECBNSS algorithm does not change much. This is because the number of active nodes under the Tian D algorithm increases linearly with the total number of nodes, resulting in a certain amount of energy waste. The network survival time is reduced. Table 3 gives the statistical data of network coverage efficiency under two algorithms.

As can be seen from Table 3, with the increase of the total number of nodes, the network coverage efficiency of ECBNSS algorithm is significantly increased than that of Tian D algorithm. It can be seen that, when the total number of nodes increases, the ECBNSS algorithm can show its superior performance.

Table 3. Comparison of network coverage efficiency

\begin{tabular}{|l|l|c|c|c|c|c|}
\hline \multicolumn{2}{|l|}{ Total number of nodes n } & 100 & 150 & 200 & 250 & 300 \\
\hline $\begin{array}{l}\text { Number of } \\
\text { active nodes }\end{array}$ & Tian D algorithm & 0.1372 & 0.1115 & 0.0892 & 0.0718 & 0.0638 \\
\cline { 2 - 7 } & ECBNSS algorithm & 0.3265 & 0.3183 & 0.3156 & 0.3091 & 0.3067 \\
\hline Multiples of increase & 1.38 & 1.85 & 2.53 & 3.31 & 3.81 \\
\hline
\end{tabular}

\section{Conclusions}

The research status of wireless sensor networks is introduced. The research on coverage control in wireless sensor networks is summarized. Through the brief introduction of the sleep mechanism, the existing problems are analyzed. On this basis, in order to optimize the network structure, a sleeping mechanism for wireless sensor networks is designed. It can better control the number of active nodes and prolong the network lifetime. The density control of the network before redundant node judgment is proposed, which avoids the uneven distribution of active nodes and the complexity of algorithm communication. A method for judging the boundary nodes and nonboundary nodes is proposed, which solves the problem that the boundary nodes cannot sleep. The simulation results show that this method makes the boundary node sleep well. The network has fewer active nodes. The nodes are more evenly distributed. The survival time of the network is extended. At the same time, it can maintain the original coverage of the network. Due to the limited research time and ability, the research work of this paper still exists many immature places. In the future work, it needs further improvement.

\section{References}

[1] Agarwal, D., \& Kishor, N. (2014). Network lifetime enhanced tri-level clustering and routing protocol for monitoring of offshore wind farms. IET Wireless Sensor Systems, 4(2), 69-79. https://doi.org/10.1049/iet-wss.2013.0050 
[2] Chanak, P., Banerjee, I., \& Sherratt, R. S. (2017). Energy-aware distributed routing algorithm to tolerate network failure in wireless sensor networks. Ad Hoc Networks, 56, 158172. https://doi.org/10.1016/j.adhoc.2016.12.006

[3] Feng, B., Tang, W., \& Guo, G. (2017). Searching Algorithm of Dormant Node in Wireless Sensor Networks. International Journal of Online Engineering, 13(5). https://doi.org/10.3991/ijoe.v13i05.7054

[4] Han, K., Luo, J., Liu, Y., \& Vasilakos, A. V. (2013). Algorithm design for data communications in duty-cycled wireless sensor networks: A survey. IEEE Communications Magazine, 51(7), 107-113. https://doi.org/10.1109/MCOM.2013.6553686

[5] Haque, M. E., Hannan, M. A., Islam, M. R., \& Rahman, M. H. H. (2016). Investigations optimum scheduling and TCP mechanism of hybrid topology sensor network in building SHM. Optik-International Journal for Light and Electron Optics, 127(6), 3218-3224. https://doi.org/10.1016/j.ijleo.2015.11.118

[6] Pazzi, R. W., Boukerche, A., De Grande, R. E., \& Mokdad, L. (2017). A clustered trailbased data dissemination protocol for improving the lifetime of duty cycle enabled wireless sensor networks. Wireless Networks, 23(1), 177-192. https://doi.org/10.1007/s11276015-1089-7

[7] Song, Y., Chen, T., Feng, Y., \& Dong, Z. (2014). Reliability Research and Analysis of Sink Node in Wireless Sensor Networks. Sensors \& Transducers, 162(1), 94.

[8] Wu, S., Niu, J., Chou, W., \& Guizani, M. (2016). Delay-Aware Energy Optimization for Flooding in Duty-Cycled Wireless Sensor Networks. IEEE Transactions on Wireless Communications, 15(12), 8449-8462. https://doi.org/10.1109/TWC.2016.2615296

[9] Yu, Y., Han, F., Bao, Y., \& Ou, J. (2015). A study on data loss compensation of WiFibased wireless sensor networks for structural health monitoring. Sensors, 13597(R2), 1.

[10] Zhang, Z., Wang, Y., Song, F., \& Zhang, W. (2015). An energy-balanced mechanism for hierarchical routing in wireless sensor networks. International Journal of Distributed Sensor Networks, 11(10), 123521. https://doi.org/10.1155/2015/123521

\section{$7 \quad$ Authors}

Ling Yang, Xucan Cai and Chunlin Wu are with School of Information Science and Technology, Zhongkai University of Agriculture and Engineering, Guangzhou, China.

Article submitted 03 December 2017. Published as resubmitted by the authors 18 January 2018. 